\title{
QUALIDADE DE VIDA E SONO DE COLABORADORES EM DIFERENTES TURNOS DE TRABALHO
}

Vanessa Cristiane Wazlawick

Universidade Regional de Blumenau

Amanda Tamanini de Araujo

Recebido em: 17/07/2020

Universidade Regional de Blumenau

Gabriela Frischknecht Petters

$1^{a}$ revisão em: 08/06/2021

Universidade Regional de Blumenau

Aceito em: 15/08/2021

\section{RESUMO}

Este estudo objetivou examinar os níveis de qualidade de vida e sono de trabalhadores que atuam em diferentes turnos em uma empresa de tecnologia do Vale do Itajaí. Participaram 159 colaboradores que responderam ao Questionário de Qualidade de Vida e ao Índice Pittsburgh de Qualidade de Sono. Os resultados apontaram correlações estatisticamente significantes positivas, embora muito fracas, entre período de trabalho diurno, percepção geral de qualidade de vida e qualidade de sono; e entre percepção de qualidade de vida e qualidade de sono. Verificou-se que $67,7 \%$ dos participantes praticam exercícios e que a percepção geral de qualidade de sono é significativamente maior neste grupo, em comparação ao que não pratica. Sugere-se que a qualidade de sono pode estar prejudicada pelo horário de trabalho e pela quantidade de horas dormidas. A prática de exercícios pode ser uma alternativa para melhora da qualidade de sono e da percepção da qualidade de vida.

Palavras-chave: sono; qualidade de vida; jornada de trabalho em turnos; saúde do trabalhador. 


\section{QUALITY OF LIFE AND SLEEP OF EMPLOYEES IN DIFFERENT SHIFT WORK SCHEDULES}

\section{ABSTRACT}

This study aimed to examine the quality of life and sleep levels of workers working on different shifts in a technology company in Vale do Itajaí. There were 159 employees, that answered the World Health Organization Quality of Life assessment and the Pittsburgh Sleep Quality Index. Results showed positive and significant correlations (although very weak), between daytime work, general perception of quality of life and sleep quality. The same was found between perception of quality of life and sleep quality. It was found that $67.7 \%$ of the participants do sports and that the general perception of sleep quality is significantly higher in this group, compared to those who do not work out. It is suggested that quality of sleep may be affected by working time and the number of hours slept. However, to practice sports may be an alternative to improve sleep quality and perception of quality of life.

Keywords: sleep; quality of life; shift work schedule; occupational health.

\section{CALIDAD DE VIDA Y SUEÑO DE EMPLEADOS EN DIFERENTES HORARIOS DE TRABAJO POR TURNOS}

\section{RESUMEN}

Este estudio buscó examinar la calidad de vida y del sueño de trabajadores que laboran en diferentes turnos en empresa de tecnología en Vale do Itajaí. Participaron 159 empleados que respondieron el Cuestionario de Calidad de Vida y el Índice de Calidad del Sueño de Pittsburgh. Los resultados mostraron correlaciones positivas significativas, aunque muy débiles, entre el período de trabajo diurno, la percepción general de la calidad de vida y del sueño; y entre percepción de calidad de vida y del sueño. Se encontró que el $67,7 \%$ de los participantes practican ejercicios y que la percepción general de la calidad del sueño de ellos es significativamente mayor que el otro grupo. Se sugiere que la calidad del sueño puede verse afectada por la cantidad de horas dormidas y trabajadas. La práctica de ejercicios puede ser una alternativa para mejorar el sueño y la percepción de la calidad de vida.

Palabras clave: sueño; calidad de vida; horario de trabajo por turnos; salud laboral. 


\section{INTRODUÇÃO}

O sono é um estado fisiológico cíclico, no qual ocorrem processos neurobiológicos fundamentais para a manutenção da saúde física e cognitiva (Bertolazi, 2008). Em um estudo brasileiro de base populacional, 29,1\% da amostra autoavaliaram seu sono como ruim, evidenciando a elevada prevalência de sono de má qualidade na população adulta (Barros, Lima, Ceolim, Zancanella \& Cardoso, 2019).

Embora os distúrbios do sono sejam frequentes na população, eles são pouco identificados. Cerca de um terço da população geral se queixa de insônia, mas menos de $20 \%$ dos casos são diagnosticados e tratados adequadamente. A sonolência excessiva aparece em até $26 \%$ da população e menos de $10 \%$ dos casos são diagnosticados e tratados corretamente. Em casos mais graves, as disfunções do sono podem causar problemas de concentração e memória e aumentar o risco de acidentes rodoviários, ocupacionais ou domésticos (Ohayon, 2011).

As altas prevalências de insônia e sonolência excessiva indicam que os distúrbios do sono são um problema de saúde pública, justificando a importância de maior atenção das autoridades de saúde (Ohayon, 2011). Estudos evidenciaram correlações significativas entre a má qualidade do sono e sintomas de depressão, ansiedade e estresse em amostras de estudantes portugueses (Galvão, Pinheiro, Gomes \& Ala, 2017; Pinto et al., 2012), demonstrando relações entre saúde mental e sono e a importância de dedicar atenção à qualidade do sono desde a fase de formação profissional.

$\mathrm{Na}$ sociedade atual, serviços essenciais, como saúde e segurança pública funcionam integralmente durante o dia e a noite, caracterizando uma sociedade 24 horas alerta (Moreno, Fischer \& Rotenberg, 2003). Este modelo é adotado em grandes indústrias e setores de tecnologia, colaborando para o aumento das jornadas de trabalho em turnos. O termo "jornada de trabalho em turnos" caracteriza o horário de trabalho em que as horas de trabalho se desviam das horas padrão, como o turno da noite e o turno rotativo (Decs, 2017). Ao longo do presente artigo, será adotada a nomenclatura trabalho em turnos não convencionais para se referir à categoria descrita.

No Manual Diagnóstico e Estatístico de Transtornos Mentais/DSM-5 (American Psychiatric Association, 2014), encontramos o transtorno do sono tipo trabalho em turnos dentro dos transtornos do sono-vigília (DSM 307.45/G47.26). Segundo o manual, o diagnóstico consiste principalmente em indivíduos que trabalham em horários regulares fora da janela diurna de 8 às 18 horas. Os sintomas predominantes que o caracterizam são sonolência excessiva no trabalho e sono prejudicado em casa de forma contínua. Geralmente estes sintomas tendem a desaparecer quando o indivíduo volta ao trabalho regular no turno diurno.

Destacam-se as consequências funcionais do transtorno, como baixo desempenho profissional e risco de sofrer acidentes no trajeto de retorno para casa. Além disso, 
estes indivíduos correm o risco de sofrerem outros prejuízos na saúde mental. como transtornos por uso de álcool ou outras substâncias e depressão, e consequências na saúde física, como distúrbios gastrintestinais, doença cardiovascular, diabetes e câncer. Frequentemente, o transtorno poderá ser um fator de risco, também, para problemas interpessoais (American Psychiatric Association, 2014).

Drake, Roehrs, Richardson, Walsh e Roth (2004) sugerem que a prevalência de transtorno do sono tipo trabalho em turnos seja de aproximadamente $10 \%$ em trabalhadores do turno noturno e em trabalhadores de turnos rotativos. Neste cenário, pesquisas com trabalhadores de diferentes turnos têm sido realizadas (Silva et al, 2019; Yazdi, Sadeghniiat-Haghighi, Loukzadeh, Elmizadeh \& Abbasi, 2014; Prata \& Silva, 2013).

Em um estudo com dois grupos de trabalhadores iranianos, a prevalência de insônia, sonolência diurna e má qualidade de sono foram significantemente maiores nos trabalhadores de turnos não convencionais quando comparados aos trabalhadores diurnos (Yazdi et al, 2014). Prata e Silva (2013) indicaram que trabalhadores do turno noturno de uma empresa industrial de Portugal manifestaram maiores queixas em relação ao sono quando comparados a trabalhadores dos turnos matutino, vespertino e alternado. Silva e colaboradores (2019) demonstraram que a presença de sonolência diurna, de distúrbios do sono e a baixa duração do sono foram fatores que contribuíram para a diminuição da qualidade do sono de profissionais de enfermagem em jornadas de trabalho de 12 horas diárias no Distrito Federal.

A qualidade do sono afeta, também, a percepção de qualidade de vida, uma vez que problemas cardíacos, hipertensão, diabetes, acidente vascular cerebral, entre outros problemas de saúde podem estar associados a distúrbios do sono (Olimpio, Trevizan, Dekon, Carvalho \& Okida, 2016). WHOQOL Group (1998) define qualidade de vida como a percepção do indivíduo sobre aspectos atuais da sua vida em relação à cultura e sistema de valores, considerando seus objetivos, expectativas, padrões e preocupações. Esse conceito refere-se a uma avaliação subjetiva embasada em um contexto cultural, ambiental e social. Engloba os domínios físico, psicológico, das relações sociais e do ambiente, este último aborda características como segurança física e proteção, recursos financeiros, transporte, oportunidades de recreação e lazer no contexto de vida do indivíduo.

Para investigar associações entre o trabalho por turnos e a qualidade de vida, um estudo croata com 1.124 enfermeiras utilizou o instrumento de avaliação da qualidade de vida WHOQOL-Bref. Os resultados não indicaram evidências de associação significativa entre trabalho por turno e qualidade de vida (Sorić, Golubić, Milosević, Juras \& Mustajbegović, 2013). Em contrapartida, uma pesquisa coreana mostrou maior risco de transtorno por uso de álcool e menores níveis de percepção de qualidade de vida em trabalhadores noturnos, em comparação com trabalhadores diurnos, especialmente entre as mulheres. Complementarmente, 
sugeriu que mulheres podem ser mais sensíveis do que homens quanto às consequências do trabalho noturno (Pham \& Park, 2019).

Relações entre a qualidade do sono e a qualidade de vida de profissionais na área da saúde também tem sido mapeada, considerando aspectos referentes aos turnos de trabalho. Viana et al. (2019) indicaram que enfermeiros do turno diurno apresentaram qualidade de vida e de sono melhores, em comparação aos que atuam no turno noturno. Guerra, Oliveira, Terreri e Len (2016) apontaram que profissionais da enfermagem possuem má qualidade de sono e sonolência diurna excessiva para os três turnos investigados (manhã/tarde/noite), porém não apresentam escores baixos para percepção de qualidade de vida, evidenciando a importância da adaptação ao turno de trabalho e da satisfação profissional.

A nível internacional, uma pesquisa grega com profissionais da saúde confirmou que o trabalho em turnos irregulares pode prejudicar as características do sono e a qualidade de vida de funcionários. Os participantes que trabalhavam em turnos não convencionais exibiram pontuação estatisticamente mais baixa no instrumento de bem-estar e qualidade de vida do que aqueles que não trabalhavam nestes períodos (Nena et al., 2018).

Quanto ao setor de trabalho que compõe esta pesquisa, Pereira (2017) objetivou analisar a relação entre as condições de trabalho e qualidade de vida de profissionais da área da tecnologia da informação $(n=170)$. A partir de dados obtidos pelo WHOQOL-Bref, constatou-se que os participantes apresentaram boa qualidade de vida, com destaque ao domínio físico. O domínio relações sociais foi o que teve a menor pontuação. A pesquisa também mostrou que as circunstâncias que mais influenciam na qualidade de vida dos profissionais foram: flexibilidade, jornada e ambiente de trabalho (Pereira, 2017).

Diante do cenário apresentado, este estudo objetivou examinar os níveis de qualidade de vida e sono de trabalhadores que atuam em diferentes turnos em uma empresa de tecnologia do Vale do Itajaí. Durante a pesquisa, verificou-se escassez de estudos científicos sobre o trabalho em turnos de profissionais da área de tecnologia. Sobre este público, foram encontrados estudos abordando qualidade de vida (Pereira, 2017), qualidade de vida no trabalho (QVT) (Madureira, Hora, \& Vieira, 2009; Silva, Volpato, \& Ronsani, 2018); QVT e estresse ocupacional (Daltro, Matos, Braz \& Nery, 2017; Silva \& Laat, 2016) e QVT e satisfação profissional (Melo, 2011). Investigar a qualidade do sono e a qualidade de vida de trabalhadores que atuam em diferentes turnos na área da tecnologia contribui com a produção científica sobre a saúde mental neste contexto. Especialmente, ao considerar que hábitos saudáveis no estilo de vida podem auxiliar na regulação dos processos psicológicos que compõem o escopo de investigação do presente estudo. 


\section{MÉTODO}

Trata-se de uma pesquisa de campo que se caracteriza como transversal descritivoexploratória com abordagem quantitativa de dados. O projeto de pesquisa foi encaminhado para avaliação do Comitê de Ética em Pesquisas com Seres Humanos da Universidade Regional de Blumenau (FURB) e teve início após a sua aprovação (No CAAE 22828919.9.0000.5370).

\section{PARTICIPANTES}

Foi adotado como critério de inclusão de sujeitos da pesquisa colaboradores maiores de dezoito anos de uma empresa de tecnologia de Santa Catarina que trabalham em diferentes turnos. Os critérios de exclusão foram: colaboradores menores de dezoito anos, colaboradores com menos de um mês de trabalho na empresa e participantes que não tenham feito a leitura integral e assinatura do Termo de Consentimento Livre e Esclarecido (TCLE).

Foram analisados os questionários de 159 participantes, 104 homens (65,4\%) e 55 mulheres (34,6\%). O grupo apresentou idades entre 18 e 64 anos, com média de 28,14 anos ( $D P=8,05$ ) e o tempo médio de trabalho na empresa foi de 3,5 anos $(\mathrm{DP}=3,04)$. A caracterização dos participantes pode ser visualizada na Tabela 1.

Tabela 1.

\section{Caracterização dos participantes}

\begin{tabular}{lcc} 
& \multicolumn{2}{c}{ Participantes } \\
\cline { 2 - 3 } & Variáveis & $\mathbf{n}$ \\
\hline Escolaridade & 9,4 & 15 \\
Ensino Médio Completo & 43,4 & 69 \\
Ensino Superior Incompleto & 33,3 & 53 \\
Ensino Superior Completo & 13,2 & 21 \\
Pós-Graduação Lato Sensu & 0,6 & 1 \\
Mestrado & & \\
Estado Civil & 52,8 & 84 \\
Solteiros & 33,3 & 53 \\
Casados/União Estável & 11,9 & 19 \\
Companheiro fixo na mesma residência & 1,9 & 3 \\
Separados/Divorciados & & \\
Possui outro emprego & 8,2 & 13 \\
Sim & 91,8 & 145 \\
Não &
\end{tabular}


Tabela 1.

\section{Continuação}

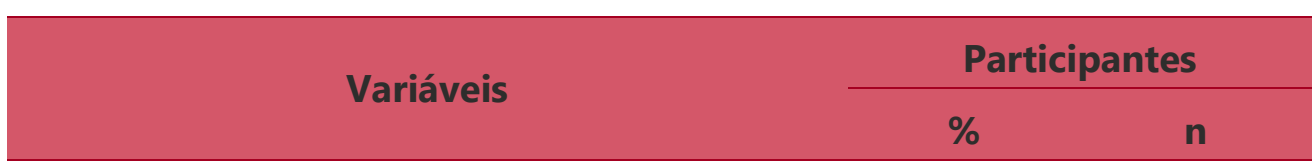

Prática de Exercício Físico

Sim

67,7

107

Não

32,3

51

Consumo de bebidas energéticas

Sim

85,5

Não

Tipo de bebida energética

Somente café

Somente chás

$3,0 \quad 4$

Somente bebida energética industrializada

$6,7 \quad 9$

Dois tipos de bebidas entre o café, chá e

15,6

\section{INSTRUMENTOS}

Neste estudo utilizaram-se três questionários: a versão reduzida do instrumento de avaliação de qualidade de vida intitulada WHOQOL-Bref (Fleck et al., 2000); o instrumento de avaliação de qualidade do sono, intitulado de Índice de Qualidade de Sono de Pittsburgh (Bertolazi, 2008); e o questionário sociodemográfico.

\section{QUESTIONÁRIO SOCIODEMOGRÁFICO}

Questionário elaborado especificamente para esta pesquisa. Investigou variáveis de perfil como: idade, sexo, escolaridade, estado civil, tempo de trabalho na empresa, setor de trabalho, turno de trabalho, tempo de trabalho no turno atual, horário de trabalho atual, se pratica exercício físico, se possui outro emprego e se tem o hábito de tomar café, chá ou bebida energética e se sim, qual bebida, horário, número de vezes ao dia e quantidade.

\section{WHOQOL-BREF (FLECK ET AL., 2000)}

O questionário de avaliação da qualidade de vida, WHOQOL-Bref foi desenvolvido pela necessidade de se ter um instrumento de rápida aplicação. Assim, o Grupo de Qualidade de Vida da Organização Mundial de Saúde determinou que se desenvolvesse a versão abreviada do WHOQOL-100, o WHOQOL-Bref. 
O instrumento é composto por 26 questões autoaplicadas, divididas em quatro domínios: físico, psicológico, relações sociais e meio ambiente. O índice alfa de Cronbach indicado para consistência interna do questionário é de $\alpha=0,91$. As respostas do questionário seguem uma escala do tipo Likert, de 1 a 5 , no qual quanto maior a pontuação, melhor a percepção de qualidade de vida. Considerase o instrumento muito útil para estudos no qual se propõem a investigar os níveis de qualidade de vida dos sujeitos, isso se dá por meio da praticidade de aplicação e compreensão. Durante a aplicação o sujeito deve circular o número que melhor representa o que está sendo perguntado (Fleck et al., 2000).

\section{ÍNDICE DE QUALIDADE DE SONO DE PITTSBURGH (BERTOLAZI, 2008)}

O questionário de avaliação da qualidade de sono, intitulado de Índice de Qualidade de Sono de Pittsburgh (PSQI-BR), foi desenvolvido por Buysse, Reynolds, Monk, Berman e Kupfer (1989) e adaptado para versão brasileira por Bertolazi (2008), avalia a qualidade do sono do sujeito em relação ao último mês. Tem por objetivo fornecer uma medida padronizada de qualidade do sono, sendo o instrumento de fácil interpretação e resposta. Assim, pelo instrumento ser considerado simples, de fácil entendimento e preenchimento rápido, é amplamente utilizado (Bertolazi, 2008).

O PSQI-BR é composto por 19 questões autoaplicadas e cinco questões respondidas por companheiros de quarto. As cinco últimas são utilizadas apenas para informação clínica e, por isso, foram retiradas da coleta de dados neste estudo. As 19 questões são agrupadas em sete componentes, com pesos distribuídos numa escala de 0 a 3, indicando que quanto maior o escore, pior a qualidade de sono do participante. Os componentes do PSQI-BR são: a qualidade subjetiva do sono, a latência para o sono, a duração do sono, a eficiência habitual do sono, os transtornos do sono, o uso de medicamentos para dormir e a disfunção diurna. O índice alfa de Cronbach de consistência interna para o questionário é de $\alpha=0,82$ (Bertolazi, 2008).

\section{PROCEDIMENTOS DE COLETA DE DADOS}

Durante toda a pesquisa, foram atendidos os fundamentos éticos indicados na Resolução 466 de 12 de dezembro de 2012. Após receberem informações pertinentes ao estudo, os participantes leram e assinaram o Termo de Consentimento Livre e Esclarecido e, em seguida, preencheram os instrumentos de coleta de dados de modo autoaplicado. Ressalta-se que a participação dos trabalhadores foi voluntária e seus dados individuais foram e continuam mantidos em sigilo. As coletas dos dados foram realizadas na empresa, em horários definidos pelo local, para que não afetasse a rotina de trabalho e que abrangesse trabalhadores dos diferentes turnos. 


\section{ANÁLISE DE DADOS}

Os dados foram tabulados e analisados com o programa IBM SPSS Statistics 22. As variáveis sociodemográficas foram analisadas por meio de estatística descritiva. A normalidade das variáveis do estudo foi verificada pelo teste de KolmogorovSmirnov. De acordo com a distribuição das variáveis, as associações entre proporção de horas trabalhadas em cada turno, variáveis de qualidade de vida e de sono foram verificadas por correlação de Spearman. Dadas as características de perfil, complementarmente, comparações de qualidade de vida e de sono entre grupos que praticam e que não praticam exercícios físicos regularmente foram realizadas por Teste $U$ de Mann-Whitney, adotando-se nível de significância $p<$ 0,05 .

Sobre a variável turno de trabalho, as respostas dos participantes indicaram variações individuais de horário de início e fim da jornada de trabalho, impossibilitando a classificação dos participantes em grupos independentes de trabalhadores diurnos e noturnos. Por isso, as variáveis referentes aos turnos de trabalho foram calculadas pela proporção de horas trabalhadas em cada um dos turnos. Para tal, foi utilizado como parâmetro a indicação da legislação brasileira da Consolidação das Leis do Trabalho, pela qual é considerado trabalho noturno o trabalho executado entre as 22 horas de um dia e as 5 horas do dia seguinte (Presidência da República, 1943). Para o cálculo da variável referente à qualidade de sono, utilizou-se a média das respostas apresentadas às questões quantitativas do questionário aplicado para este construto.

\section{RESULTADOS}

O presente estudo foi norteado pela pergunta de pesquisa: "quais as relações entre a proporção de tempo no trabalho diurno/noturno, qualidade de sono e qualidade de vida de colaboradores de uma empresa de tecnologia?". A Tabela 2 apresenta os resultados referentes às correlações entre as variáveis de turno de trabalho, de qualidade de vida e qualidade de sono.

Tabela 2.

Correlações entre Tempo de trabalho, Sono e Qualidade de Vida

\begin{tabular}{|c|c|c|c|c|c|c|c|c|c|}
\hline & $\begin{array}{l}\text { Trab. } \\
\text { notur. }\end{array}$ & $\begin{array}{l}\text { Trab. } \\
\text { diurno }\end{array}$ & $\begin{array}{l}\text { Horas } \\
\text { sono }\end{array}$ & $\begin{array}{c}\text { Qualid.s } \\
\text { ono }\end{array}$ & $\begin{array}{l}\text { QV } \\
\text { Físico }\end{array}$ & QV Psic. & $\begin{array}{l}\text { QV Rel. } \\
\text { Sociais }\end{array}$ & $\begin{array}{l}\text { QV } \\
\text { Amb. }\end{array}$ & $\begin{array}{l}\text { QV } \\
\text { Geral }\end{array}$ \\
\hline $\begin{array}{l}\text { Trabalho } \\
\text { noturno }\end{array}$ & 1,000 & & & & & & & & \\
\hline $\begin{array}{l}\text { Trabalho } \\
\text { diurno }\end{array}$ & $-1,000^{\star *}$ & 1,000 & & & & & & & \\
\hline $\begin{array}{l}\text { Horas de } \\
\text { sono }\end{array}$ & $-0,24^{* *}$ & $0,24^{* *}$ & 1,000 & & & & & & \\
\hline $\begin{array}{l}\text { Qualid. } \\
\text { sono }\end{array}$ & $-0,21^{* *}$ & 0,21 ** & 0,21 * & 1,000 & & & & & \\
\hline QV Físico & $-0,15$ & 0,15 & 0,15 & 0,23 ** & 1,000 & & & & \\
\hline
\end{tabular}


Tabela 2.

\section{Continuação}

\begin{tabular}{|c|c|c|c|c|c|c|c|c|c|}
\hline & $\begin{array}{l}\text { Trab. } \\
\text { notur. }\end{array}$ & $\begin{array}{l}\text { Trab. } \\
\text { diurno }\end{array}$ & $\begin{array}{c}\text { Horas } \\
\text { sono } \\
\end{array}$ & $\begin{array}{c}\text { Qualid.s } \\
\text { ono }\end{array}$ & $\begin{array}{c}\text { QV } \\
\text { Físico }\end{array}$ & QV Psic. & $\begin{array}{l}\text { QV Rel. } \\
\text { Sociais }\end{array}$ & $\begin{array}{c}\text { QV } \\
\text { Amb. }\end{array}$ & $\begin{array}{c}\text { QV } \\
\text { Geral }\end{array}$ \\
\hline QV Psic. & $-0,16 *$ & $0,16^{*}$ & 0,11 & $0,19 *$ & $0,23^{\star *}$ & 1,000 & & & \\
\hline $\begin{array}{l}\text { QV Rel. } \\
\text { Sociais }\end{array}$ & $-0,15$ & 0,15 & 0,03 & 0,13 & $0,19 *$ & $0,58^{\star *}$ & 1,000 & & \\
\hline QV Amb. & $-0,08$ & 0,08 & 0,1 & 0,21 ** & 0,13 & $0,49 * *$ & $0,45^{\star *}$ & 1,000 & \\
\hline QV Geral & $-0,16 *$ & $0,16^{*}$ & 0,110 & $0,23 * *$ & $0,21^{\star \star}$ & $0,83^{* *}$ & $0,7^{\star *}$ & $0,76^{\star \star}$ & 1,000 \\
\hline
\end{tabular}

${ }^{*} \mathrm{p}<0,05$

${ }^{* *} \mathrm{p}<0,01$

Na Tabela 2, observa-se que há uma correlação positiva, embora muito fraca, entre a proporção de tempo que o colaborador trabalha no período diurno e a sua qualidade de sono $(r=0,21 ; p<0,01)$. Da mesma forma, observa-se uma correlação negativa, também muito fraca, entre proporção de tempo que o colaborador trabalha no período noturno e a sua qualidade de sono $(r=-0,21 ; p<0,01)$. Isto pode sugerir que quanto mais horas o colaborador trabalha no período noturno pior pode a ser a sua qualidade de sono.

No que diz respeito às correlações entre turno de trabalho e qualidade de vida (e seus domínios), verifica-se correlação positiva, embora muito fraca, entre a proporção de tempo que o colaborador trabalha no período diurno e a sua qualidade de vida psicológica $(r=0,16 ; p<0,05)$. Da mesma maneira, observa-se uma correlação negativa e muito fraca entre a proporção de tempo de trabalho no período noturno e a sua qualidade de vida psicológica $(r=-0,16 ; p<0,05)$. Isso nos mostra que, quanto mais horas o colaborador trabalha no período noturno pior pode a ser a sua percepção de qualidade de vida ligada à saúde psicológica.

Percebe-se, ainda, uma correlação positiva, ainda que muito fraca, entre a proporção de tempo que o colaborador trabalha no período diurno e a sua qualidade de vida geral $(r=0,16 ; p<0,05)$. Assim como, uma correlação negativa e muito fraca entre a proporção de tempo que o colaborador trabalha no período noturno e a sua qualidade de vida geral $(r=-0,16 ; p<0,05)$. Portanto, os resultados indicam que, quanto mais horas o colaborador trabalha no período noturno pior tende a ser a sua percepção geral de qualidade de vida. Não foram verificadas correlações estatisticamente significantes $(p>0,05)$ entre a proporção de horas de trabalho nos períodos noturno e diurno e os demais domínios de qualidade de vida (físico; relações sociais; ambiente).

Para além dos pontos mencionados, verificou-se que o período de trabalho diurno/noturno também interfere no tempo (horas) de sono dos colaboradores, com uma correlação positiva e muito fraca $(r=0,24 ; p<0,01)$. Este ponto chama a atenção na medida em que a quantidade de horas de sono também parece interferir na qualidade do sono, com uma correlação positiva e muito fraca entre estas variáveis $(r=0,21 ; p<0,05)$. Este dado pode indicar que, de certa forma, a qualidade de sono pode estar sendo prejudicada tanto pelo horário de trabalho 
quanto pela quantidade de horas dormidas pelos colaboradores. Estes pontos se tornam importantes ao considerar que a qualidade do sono parece interferir sobre a qualidade de vida dos domínios: físico $(r=0,23 ; p<0,01)$, psicológico $(r=0,19 ; p$ $<0,05)$, ambiente $(r=0,21 ; p<0,01)$ e geral $(r=0,23 ; p<0,01)$, ainda que por uma correlação muito fraca.

Uma característica de perfil que chama a atenção se refere ao estilo de vida ativo do referido grupo de participantes. Conforme visto, 67,7\% do grupo pratica exercícios físicos regularmente. A partir disso, foram comparados os níveis de qualidade de sono e de qualidade de vida entre grupos de participantes que praticam e que não praticam exercícios físicos. A Tabela 3 demonstra que o grupo de praticantes de exercícios físicos apresentou médias mais elevadas de qualidade do sono, bem como de qualidade de vida, exceto para o domínio qualidade de vida nas relações sociais. Contudo, somente a comparação para a qualidade de sono indicou uma diferença estatisticamente significante $(p<0,05)$.

Tabela 3.

\section{Comparação das médias de Qualidade de Vida e Qualidade do Sono por grupo}

\begin{tabular}{lccccc}
\hline \multirow{2}{*}{ Variáveis } & \multicolumn{2}{c}{$\begin{array}{c}\text { Praticantes de } \\
\text { exercícios }\end{array}$} & \multicolumn{2}{c}{$\begin{array}{c}\text { Não praticantes de } \\
\text { exercícios }\end{array}$} & $\begin{array}{c}\text { Significância } \\
\text { Estatística }\end{array}$ \\
\cline { 2 - 6 } & Média & DP & Média & DP & p \\
\hline QV Físico & 14,95 & 2,16 & 14,67 & 2,26 & $\mathrm{p}>0,05$ \\
QV Psicológico & 14,25 & 2,50 & 13,75 & 2,32 & $\mathrm{p}>0,05$ \\
QV Rel. Sociais & 14,41 & 2,88 & 14,54 & 3,12 & $\mathrm{p}>0,05$ \\
QV Ambiente & 14,44 & 1,9 & 14,4 & 2,00 & $\mathrm{p}>0,05$ \\
QV Geral & 14,54 & 1,85 & 14,32 & 1,90 & $\mathrm{p}>0,05$ \\
Qualidade Sono & $\mathbf{2 , 1 2}$ & $\mathbf{0 , 4 2}$ & $\mathbf{1 , 9 5}$ & $\mathbf{0 , 5 0}$ & $\mathbf{p}<\mathbf{0 , 0 5 *}$ \\
\hline
\end{tabular}

* Diferença estatisticamente significante entre as médias dos grupos (Teste $U$ de Mann-Whitney)

Os resultados nos mostram diferenças estatisticamente significantes na qualidade do sono dos colaboradores nos diferentes turnos de trabalho. Cada ponto que se mostrou significativo será discutido com o suporte da literatura na próxima seção. Os dados apresentados nos sugerem, ainda que de modo preliminar, que a prática de exercícios físicos regulares pode, de algum modo, auxiliar na melhora e manutenção da qualidade de sono e, consequentemente, da qualidade de vida dos indivíduos.

\section{DISCUSSÃO}

Este estudo teve como objetivo examinar os níveis de qualidade de vida e sono de trabalhadores que atuam em diferentes turnos em uma empresa de tecnologia do Vale do Itajaí. No estudo de Viana et al. (2019) os enfermeiros que trabalhavam em 
turno diurno também mostraram melhor qualidade de sono e qualidade de vida em comparação aos trabalhadores do turno noturno. Prata e Silva (2013), em seu estudo com trabalhadores de uma indústria eletrônica em Portugal, também obtiveram como resultados que, aqueles que trabalham algum período da sua jornada no período noturno apresentaram mais problemas de sono quando comparados aos que atuam em outros turnos. Estes dados corroboram os resultados encontrados no presente estudo, em que a qualidade de sono dos colaboradores que trabalham maior proporção de tempo no período noturno apresentou-se como significativamente inferior em comparação aos colaboradores que trabalham maior proporção de tempo no período diurno. Considera-se que estratégias de avaliação e intervenção que facilitem a organização de rotina e de hábitos ligados ao sono dos colaboradores que trabalham no turno noturno poderiam contribuir com a percepção de qualidade de sono, importante para o desempenho geral de atividades diárias, inclusive no trabalho.

Em relação à proporção de trabalho no período diurno/noturno e à qualidade de vida no domínio psicológico, os resultados sugerem que quanto mais horas o colaborador trabalha no período noturno, menor tende a ser a sua percepção de qualidade de vida. Assim como mostra a pesquisa de Viana et al. (2019) em que, no trabalho de turno diurno os profissionais que apresentaram boa qualidade do sono apresentam melhor qualidade de vida no domínio psicológico. Ainda que seja clara a relação entre diversos fatores com a percepção de qualidade de vida, ao considerar as associações entre qualidade de vida e sono encontradas neste estudo, sugere-se que processos de intervenção voltados à saúde do trabalhador para melhora da qualidade de sono possam, ainda que de forma indireta, contribuir também com a sua percepção de qualidade de vida.

Em contraponto, um estudo com equipe de enfermagem noturna de um hospital universitário, Severo (2012) obteve resultados positivos relacionando qualidade de vida psicológica e trabalho noturno. Um dos entrevistados se declara bastante satisfeito com o sentido da vida, o que possibilita associar esta percepção, em um contexto ecossistêmico, tanto à "arte" do cuidado presente da enfermagem, sentindo-se útil para outro ser humano, quanto pelas relações e experiências que vivencia em seu mundo e seu viver (Severo, 2012). É importante considerar que, em nosso estudo, as correlações encontradas entre proporção de horas trabalhadas em cada turno com percepção de qualidade de vida e de sono foram muito fracas, ainda que estatisticamente significantes. É importante questionar se a classificação de trabalhadores em grupos independentes de trabalho em turno diurno e noturno traria resultados mais fortes neste sentido. Entretanto, esta separação não foi possível devido às variações individuais encontradas entre os participantes para os horários de início e fim de turno de trabalho.

Ao relacionar trabalho no turno noturno e qualidade de vida nas relações sociais, Severo (2012) aponta, ainda, que os trabalhadores estão "satisfeitos" com suas relações sociais, destacando-se a faceta de maior escore a que se situa nos amigos, parentes, conhecidos, colegas. A autora coloca que, este é um ponto positivo, pois 
demonstra solidariedade, parceria, e o trabalho conjunto compartilhado entre si no período noturno por não serem encontrados vários serviços institucionais neste período de trabalho. Sugere que estas características podem levar ao fortalecimento de outros aspectos pessoais e, também, profissionais do trabalhador (Severo, 2012). Na presente pesquisa, não foram encontradas correlações estatisticamente significantes entre os fenômenos de interesse e qualidade de vida no domínio psicológico. Ainda assim, os dados de Severo (2012) evidenciam a importância da rede de apoio e da manutenção das relações sociais, independente do horário de trabalho.

Examinou-se, também, que o período de trabalho diurno/noturno interfere no tempo (horas) de sono dos colaboradores participantes. Este dado chama atenção, uma vez que a quantidade de horas de sono parece interferir na qualidade do sono. Sugere-se que a qualidade de sono pode estar prejudicada tanto pelo horário de trabalho quanto pela quantidade de horas dormidas pelos colaboradores. Em uma pesquisa com profissionais de enfermagem de um serviço móvel de urgência, Silva et al. (2019) observaram que $56 \%$ dos trabalhadores apresentaram baixa qualidade do sono. Os autores salientam que os aspectos que mais corroboram a queda na qualidade do sono dos profissionais de enfermagem seriam a sonolência diurna, os distúrbios do sono e a duração do sono (tempo), o que sustenta o resultado desta pesquisa, pelo qual percebeu-se que, a qualidade de sono pode estar prejudicada tanto pelo horário de trabalho quanto pela quantidade de horas dormidas pelos colaboradores. Nossos dados mostraram que a qualidade do sono parece estar relacionada com a qualidade de vida nos domínios físico, psicológico, ambiente e geral. Viana et al. (2019) evidenciam, em sua amostra de enfermeiros, a associação entre o padrão do sono e a qualidade de vida, em que a percepção de qualidade de vida parece melhorar, a medida em que melhora a qualidade de sono.

Finalmente, de modo complementar, as associações entre variáveis de perfil e variáveis psicológicas demonstraram que o grupo de trabalhadores que pratica exercícios regularmente apresentou maiores médias de qualidade de sono, em comparação ao grupo que não pratica. Considerando as associações entre qualidade de vida e sono, destaca-se o estudo de Silva, Silva, Silva, Souza e Tomasi (2010), em que os autores colocam que é possível verificar que, quão mais ativa a pessoa é, melhor tende a ser sua percepção de qualidade de vida. A escassez de estudos sobre hábitos saudáveis no estilo de vida para melhora da saúde mental de colaboradores que atuam na área de tecnologia foi evidenciada durante a realização deste estudo. Este dado mostra a importância de que estudos futuros explorem esta e outras variáveis que possam facilitar os processos de adaptação e organização de rotina, especialmente na área do trabalho em diferentes turnos, no contexto da tecnologia e de outras áreas de atuação profissional.

\section{CONSIDERAÇÕES FINAIS}


Este estudo teve como objetivo examinar os níveis de qualidade de vida e sono de trabalhadores que atuam em diferentes turnos em uma empresa de tecnologia do Vale do Itajaí. Pôde-se observar que os colaboradores que trabalham em maior proporção de tempo no horário noturno tendem a relatar uma percepção pior de qualidade de vida e qualidade do sono em relação aos colaboradores que trabalham em maior proporção de tempo no horário diurno. Para colaboradores que praticam exercício físico, a qualidade do sono tende a ser melhor. Este é um ponto positivo desta pesquisa, com destaque para o fato de que a maioria dos participantes praticavam exercício físico. O estilo de vida ativo parece contribuir positivamente com a qualidade do sono destes colaboradores. Especula-se, ainda, que este dado pode favorecer a percepção de qualidade de vida de modo indireto.

O trabalho em turnos é uma possibilidade para atender as características e necessidades da sociedade atual. Para tanto, faz-se necessário elaborar estratégias de enfrentamento e adaptação ao horário de trabalho, especialmente no turno noturno. A produção científica sobre a saúde mental de profissionais da tecnologia auxilia no estabelecimento de mecanismos e estratégias para intervenções em Psicologia Organizacional e saúde do trabalhador. As possibilidades de intervenção incluem a implantação de programas de QVT, psicoeducação quanto à qualidade do sono, higiene do sono, importância e benefícios de atividades físicas regulares para melhora da saúde mental em geral. Quanto à agenda de pesquisa futura, sugere-se que sejam realizados estudos sobre qualidade de vida e qualidade do sono em profissionais da tecnologia com trabalho em turnos, com temas como: relógio biológico e horário de trabalho; trabalho noturno e desempenho no trabalho; qualidade do sono e sua relação com o nível de atividade física.

Como limitações do estudo, considera-se que os níveis fracos, ainda que estatisticamente significantes, de correlação estatística possibilitam, apenas, indicações iniciais sobre o tema. A escassez de pesquisas acerca da qualidade de vida e qualidade de sono em profissionais da área de tecnologia também foi um fator de limitação encontrado. Estudos futuros, com amostras maiores e seleção aleatória de participantes, são necessários para possibilitar o uso de procedimentos mais robustos de análise de dados, maior aprofundamento na interpretação dos resultados e, consequentemente, ampliação da produção científica neste cenário.

\section{REFERÊNCIAS}

American Psychiatric Association. (2014). Manual Diagnóstico e Estatístico de Transtornos Mentais: DSM-5 [Recurso eletrônico]. (5a ed.; M. I. C. Nascimento Trad.). Porto Alegre, RS: Artmed.

Barros, M. B. A., Lima, M. G., Ceolim, M. F., Zancanella, E., \& Cardoso, T. A. M. O. (2019). Quality of sleep, health and well-being in a population-based study. Revista de Saúde Pública, 53, 82. https://doi.org/10.11606/s1518-8787.2019053001067

Bertolazi, A. N. (2008). Tradução, Adaptação cultural e validação de dois instrumentos de avaliação do sono: Escala de sonolência de Epworth e Índice de qualidade de sono de Pittsburgh (Dissertação de mestrado). Universidade Federal do Rio Grande do Sul, Porto Alegre.

Presidência da República. Casa Civil. Subchefia para Assuntos Jurídicos. (1943). Decreto-lei $n^{\circ} 5.452$ de $1^{\circ}$ de maio de 1943. Aprova a Consolidação das Leis do Trabalho. Brasília, DF. Recuperado de http://www.planalto.gov.br/ccivil 03/decreto-lei/del5452.htm 
Daltro, M. S., Matos, R. L., Braz, H. M. F. S. \& Nery, M. B. M. (2017). O estresse ocupacional e a qualidade de vida no trabalho: um estudo sobre a percepção dos funcionários de TI no setor bancário. Entrepreneurship, 1(1), 71-82. https://doi.org/10.6008/SPC2595-4318.2017.001.0007

Descritores em Ciências da Saúde: DeCS. (2017). Ed 2017. rev. e ampl. São Paulo: BIREME /OPAS / OMS. Recuperado de http://decs.bvsalud.org

Drake, C. L., Roehrs, T., Richardson, G., Walsh, J. K., \& Roth, T. (2004). Shift Work Sleep Disorder: Prevalence and Consequences Beyond that of Symptomatic Day Workers. Sleep, 27(8), 1453 1462. https://doi.org/10.1093/sleep/27.8.1453

Fleck, M. P. A., Louzada, S., Xavier, M., Chachamovich, E., Vieira, G., Santos, L., \& Pinzon, V. (2000). Aplicação da versão em português do instrumento abreviado de avaliação da qualidade de vida "WHOQOL-Bref". Revista de Saúde Pública, 34(2), 178-183. https://doi.org/10.1590/S0034-89102000000200012

Galvão, A., Pinheiro, M., Gomes, M. J., \& Ala, S. (2017). Ansiedade, stress e depressão relacionados com perturbações do sono-vigília e consumo de álcool. Revista Portuguesa de Enfermagem de Saúde Mental, n. spe5, 8-12. http://dx.doi.org/10.19131/rpesm.0160

Guerra, P. C., Oliveira, N. F., Terreri, M. T. S. L. R. A., \& Len, C. A. (2016). Sono, qualidade de vida e humor em profissionais de enfermagem em Unidades de Terapia Intensiva Infantil. Revista da Escola de Enfermagem da USP, 50(2), 279-285. http://dx.doi.org/10.1590/S0080$\underline{623420160000200015}$

Madureira, J. L., Hora, H. R. M. \& Vieira, L. E. V. (2009). Qualidade de Vida no Trabalho: Um estudo de caso em uma empresa de tele-atendimento em Tecnologia da Informação. XVI Simpósio de Engenharia de Produção, 12.

Melo, E. R. (2011). Teletrabalho, qualidade de vida no trabalho e satisfação profissional: um estudo exploratório numa amostra de profissionais na área da tecnologia da informação (Dissertação de Mestrado). Universidade de Lisboa, Lisboa.

Moreno, C. R. C., Fischer, F. M., \& Rotenberg, L. (2003). A saúde do trabalhador na sociedade 24 horas. São Paulo em Perspectiva, 17(1), 34-46. https://doi.org/10.1590/S0102-88392003000100005

Nena, E., Katsaouni, M., Steiropoulos, P., Theodorou, E., Constantinidis, T. C., \& Tripsianis, G. (2018). Effect of Shift Work on Sleep, Health, and Quality of Life of Health-care Workers. Indian Journal of Occupational and Environmental Medicine, 22(1), 29-34. https://dx.doi.org/10.4103\%2Fijoem.IJOEM_4_18

Ohayon, M. M. (2011). Epidemiological Overview of sleep Disorders in the General Population. Sleep Med Res, 2(1), 1-9. https://doi.org/10.17241/smr.2011.2.1.1

Olimpio, R. W., Trevizan, A. C. S., Dekon, S. F. C., Carvalho, K. H. T. \& Okida, R. C. (2016). Qualidade do sono para uma melhor qualidade de vida. Anais do $7^{\circ}$ Sim Saúde - Simpósio em Saúde 2016. Archives of Health Investigation, 5. http://dx.doi.org/10.21270/archi.v5i0.1716

Pereira, M. D'A. (2007). Qualidade de vida dos profissionais de TI e as condições de trabalho (Dissertação de Mestrado). Pontifícia Universidade Católica de São Paulo, São Paulo.

Pham, T.T., \& Park, B. (2019). Alcohol use disorder and health-related quality of life in Korean nightshift workers: A cross-sectional study using the KNHANES 2007-2015 data. PLoS ONE, 14(4), Article e0214593. https://doi.org/10.1371/journal.pone.0214593

Pinto, T. R., Amaral, C., Silva, V. N., Silva, J., Leal, I. P., \& Paiva, T. (2012). Hábitos de sono e ansiedade, depressão e stresse: Que relação? Anais do 12. Colóquio Internacional de Psicologia e Educação: Educação, aprendizagem e desenvolvimento: Olhares contemporâneos através da investigação e da prática (p. 990-1006). Lisboa: ISPA - Instituto Universitário.

Prata, J., \& Silva, I. S. (2013). Efeitos do trabalho em turnos na saúde e em dimensões do contexto social e organizacional: um estudo na indústria eletrônica. Revista Psicologia Organizações e Trabalho, 13(2), 141-154. Recuperado de http://pepsic.bvsalud.org/pdf/rpot/v13n2/v13n2a04.pdf

Severo, D. F. (2012). Qualidade de vida dos trabalhadores de enfermagem do turno noturno do HU/FURG: na perspectiva ecossistêmica (Dissertação de Mestrado). Universidade Federal do Rio Grande, Rio Grande.

Silva, J. S. X., Silva, R. M., Cangussu, D. D. D., Moraes Filho, I. M., Pérez, M. A., \& Proença, M. F. R. (2019). Qualidade do sono dos profissionais de enfermagem do serviço móvel de atendimento de urgência. Revista de Divulgação Científica Sena Aires, 8(3), 264-272. https://doi.org/10.36239/revisa.v8.n3.p264a272

Silva, M. A. L. \& Laat, E. F. (2016). A hardiness pode influenciar na qualidade de vida no trabalho? um estudo com profissionais de tecnologia da informação em universidades estaduais do 
Espacios,

37(20),

23.

Recuperado

de

https://www.revistaespacios.com/a16v37n20/16372025.html

Silva, R. S., Silva, I., Silva, R. A., Souza, L., \& Tomasi, E. (2010). Atividade física e qualidade de vida. Ciência \& Saúde Coletiva, 15(1), 115-120. https://doi.org/10.1590/S141381232010000100017

Silva, T. K., Volpato, D. \& Ronsani, C. (2018). Análise da qualidade de vida no trabalho em uma empresa do setor de tecnologia da informação localizada na cidade de Criciúma-SC. Revista de Carreiras e Pessoas, 8(2), 258-281. https://doi.org/10.20503/recape.v8i2.34351

Sorić, M., Golubić, R., Milosević, M., Juras, K., \& Mustajbegović, J. (2013). Shift work, quality of life and work ability among Croatian hospital nurses. Collegium Antropologicum, 37(2), 379-384.

Viana, M., Silva, K., De Martino, M., Bezerra, C., Oliveira, A., Souza, A. M. L., ... Torres, G. (2019). Qualidade de vida e sono de enfermeiros nos turnos hospitalares. Revista Cubana De $\begin{array}{llll}\text { Enfermería, 35(2), } & \text { 1-19. } & \text { Recuperado }\end{array}$ http://www.revenfermeria.sld.cu/index.php/enf/article/view/2137

Yazdi, Z., Sadeghniiat-Haghighi, K., Loukzadeh, Z., Elmizadeh, K., \& Abbasi, M. (2014). Prevalence of Sleep Disorders and Their Impacts on Occupational Performance: A Comparison between Shift Workers and Nonshift Workers. Sleep disorders, 1-5, Article 870320 https://doi.org/10.1155/2014/870320

WHOQOL Group. (1998). Development of the World Health Organization WHOQOL-Bref Quality of Life Assessment. Psychological Medicine, 28(3), 551-558. https://doi.org/10.1017/s0033291798006667

\section{CONFLITOS DE INTERESSES}

Não há conflitos de interesses.

\section{SOBRE OS AUTORES}

Vanessa Cristiane Wazlawick é psicóloga graduada pela Universidade Regional de Blumenau (FURB). E-mail: vanessacristianew@hotmail.com

(2) https://orcid.org/0000-0003-3490-6823

Amanda Tamanini de Araujo é psicóloga graduada pela Universidade Regional de Blumenau (FURB). E-mail: ataraujo@furb.br.

(2) https://orcid.org/0000-0002-3442-0762

Gabriela Frischknecht Petters é psicóloga graduada pela Universidade Regional de Blumenau (FURB), Mestre e Doutora em Psicologia pela Universidade Federal de Santa Catarina (UFSC). Docente do Departamento de Psicologia da FURB.

E-mail: gfrischknecht@furb.br.

(1) https://orcid.org/0000-0001-5297-0998 